\title{
Análise da Participação de Pequenas Centrais Hidrelétricas no Mecanismo de Realocação de Energia
}

\author{
Gabriela M. Peixoto* Rafael B. Pereira** Luciana S. Amorim** \\ Thiago M. Machado-Coelho*** Gustavo L. Soares** Gustavo G. Parma* \\ * Departamento de Engenharia Elétrica, Pontifícia Universidade Católica de Minas Gerais, \\ Belo Horizonte-MG (email: gabi.mp@ @otmail.com; gustavo.parma@gmail.com) \\ ** Programa de Pós-Graduação em Engenharia Elétrica, Pontifícia Universidade Católica de Minas Gerais, \\ Belo Horizonte-MG (e-mail: rafael.bambirra@sga.pucminas.br; lucianamorimm@gmail.com; gsoares@pucminas.br) \\ *** Programa de Pós-Graduação em Engenharia Elétrica, Universidade Federal de Minas Gerais, \\ Belo Horizonte -MG (e-mail: thmmcoelho@ufmg.br)
}

\begin{abstract}
The objective of this work is to propose a decision-making methodology to evaluate whether Small Hydroelectric Power Plants (PCHs) should participate in the Brazilian Energy Reallocation Mechanism (MRE), and what is the best contracting process to minimize the risk of the venture. The NEWAVE model was used to optimize the systems' operation. The simulations were done in MATLAB, obtaining the financial results of the PCHs based on the parameters obtained from the NEWAVE model. The averse risk measure CVaR was then applied to evaluate the revenues at risk. Graphs with the risk profile of each plant were used to individually evaluate each intrinsic risk. Thus, the methodology proposes to lower the exposure of the power plants to the spot market, mitigating the instabilities of the MRE and the short-term market, to find the best contracts for the scenarios and to maxime revenue.
\end{abstract}

Resumo: O objetivo do trabalho é propor uma metodologia para tomada de decisão que avalia se Pequenas Centrais Hidroelétricas (PCHs) devem participar do Mecanismo de Realocação de Energia (MRE), e qual a melhor contratação para minimizar o risco dos empreendimentos. O modelo NEWAVE foi utilizado para obter séries de parâmetros para a otimização da operação dos sistemas. As simulações foram feitas em ambiente MATLAB, obtendo-se a distribuição dos resultados financeiros das PCHs, embasados nos parâmetros obtidos do processamento do NEWAVE. A métrica de aversão ao risco CVaR foi aplicada para avaliar as receitas em risco dentro e fora do MRE. Foram construídos gráficos com o perfil de risco de cada usina e cada risco intrínseco foi avaliado individualmente. Propõe-se, assim, menor exposição das usinas ao mercado spot, atenuando as instabilidades do mecanismo e do mercado de curto prazo, encontrando a melhor contratação para os cenários e maximização das receitas.

Keywords: Energy Reallocation Mechanism; Small Hydroelectric Power Plant; Generation Scaling Factor, Energy planning, risk analysis; Conditional Value at Risk

Palavras-chaves: Mecanismo de Realocação de Energia; Pequenas Centrais Hidrelétricas; Generation Scaling Factor (GSF); Planejamento energético; Análise de risco; Conditional Value at Risk.

\section{INTRODUÇÃO}

O Sistema Elétrico Brasileiro (SEB) é composto por uma matriz predominantemente hidro-termo-eólica, sendo as outras fontes complementares à matriz energética, agregando confiabilidade ao sistema (ONS, 2019). A interligação da geração proporciona o intercâmbio de energia entre os subsistemas do país e garante a segurança no atendimento às demandas do sistema elétrico, mas proporciona complexidade aos processos de operação e comercialização de energia (EPE, 2015).

O setor elétrico passou por constantes reformas estruturais envolvendo a desverticalização do setor, entretanto ainda é classificado como um sistema centralizado e regulado (Moita, 2008). O despacho das usinas hidrelétricas de grande porte é realizado de forma centralizada sob competência do Operador Nacional do Sistema (ONS) e não do proprietário do empreendimento, fator que afeta a previsibilidade do gerador e, consequentemente, a receita esperada pela contratação das usinas (CCEE, 2018).

Do ponto de vista do planejamento, a modelagem em cascata é adotada pelo ONS como forma de garantir a gestão estratégica dos armazenamentos, que pode divergir da geração ótima individual do agente gerador. Dessa forma, no caso específico dos empreendimentos hidrelétricos, foi estabelecido o Mecanismo de Realocação de Energia (MRE) com o objetivo de compartilhar o risco hidrológico entre os agentes geradores, associado à operação e comercialização das usinas de despacho centralizado pelo ONS (ANEEL, 1998).

As usinas hidrelétricas despachadas centralizadamente pelo ONS possuem participação compulsória ao MRE. Já as PCHs, apesar de serem empreendimentos hidrelétricos, não tem a participação obrigatória nesse mecanismo e podem optar por entrar ou não, no ano anterior à decisão de participar. Se 
decidirem entrar, devem permanecer por no mínimo doze meses, conforme a Resolução Normativa $n^{\circ}$ 409/2010. Portanto, é importante o estabelecimento de uma metodologia para avaliar a entrada ou não desses empreendimentos no mecanismo.

A compra e venda de energia das PCHs pode ser realizada por contratos bilaterais ou no mercado de curto prazo, sendo de responsabilidade do proprietário da usina arcar com os riscos de geração do empreendimento. Contratos bilaterais são alternativas utilizadas pelos agentes para se protegerem das oscilações de preço no Mercado de Curto Prazo (MCP) (Fanzeres, Street and Barroso, 2015). Ao lidar com esses contratos, as empresas negociam entre si um preço de compra ou venda de energia e uma validade para a operação (Street et al., 2009). Essas transações, entretanto, podem expor o gerador ao custo de oportunidade em ocasiões em que o mercado spot apresente preço superior ao firmado nos contratos (Masukawa, 2011).

O presente trabalho propõe uma metodologia para tomada de decisão em dois níveis: (a) entrar ou não entrar no MRE e (b) qual a melhor contratação para minimização do risco. Isso é possível com a implementação da métrica Conditional Value at Risk (CVAR) nas séries do modelo NEWAVE.

A análise do comportamento do MRE visa obter o deslocamento de geração do Sistema Interligado Nacional (SIN) com o intuito de proporcionar cenários base para decisões estratégicas na participação, ou não, do empreendimento no mecanismo. As análises deste estudo incluem as avaliações de risco Média e CVaR, os quais possibilitam a otimização dos cenários relativos ao desempenho das PCHs dentro do MRE visando a permanência das mesmas no mecanismo.

A metodologia proposta foi aplicada exclusivamente no caso real de quatro PCHs, localizadas no estado de Minas Gerais. As simulações necessárias foram realizadas em ambiente MATLAB.

Neste trabalho, a Seção 2 apresenta o referencial teórico e a Seção 3, a metodologia proposta. Na Seção 4 têm-se os experimentos e resultados e, por fim, na Seção 5 têm-se as conclusões.

\section{REFERENCIAL TEÓRICO}

\subsection{Planejamento do Setor Elétrico}

A política de planejamento energético no Brasil considera como premissa a sinergia entre expansão e operação para garantir a confiabilidade e economicidade do sistema (EPE, 2015). No Brasil, estas atividades são atribuídas à duas instituições do setor, a Empresa de Pesquisa Energética (EPE) e o ONS. As premissas de expansão consideram sempre a necessidade energética e os custos de implantação e operação das fontes, além dos prazos para entrada em operação dos empreendimentos contratados em futuros leilões (EPE, 2015). A implementação de projetos hidrelétricos contribui para a diversificação da matriz elétrica, além de apresentarem risco ambiental reduzido em comparação a outras fontes. Neste contexto, surge mercado favorável para as PCHs.

As PCHS se caracterizam por apresentar potência instalada de 1 a 30 MW, conforme Resolução n ${ }^{\circ} 652,2003$, normalmente estão próximas aos centros de carga e são muito utilizadas como recurso de autoprodução, figura onde o consumidor é o proprietário da usina de geração (EPE, 2015).

\subsubsection{NEWAVE}

O NEWAVE é o modelo estratégico de geração hidrotérmica a subsistemas equivalentes utilizado pelo ONS, com o objetivo de otimizar a operação a longo e médio prazo dos subsistemas hidrotérmicos que compõem a matriz de geração do SIN (CEPEL, 2013).

O NEWAVE é constituído da aplicação de políticas de operação na representação do parque hidroelétrico nacional e é utilizado no planejamento de operação do sistema elétrico como suporte a tomada de decisão para um melhor aproveitamento dos reservatórios. Além disto, o modelo é utilizado na metodologia de cálculo do preço de liquidação das diferenças (PLD) pela Câmara de Comercialização de Energia Elétrica (CCEE) (EPE, 2015).

O processamento do modelo NEWAVE retorna 2000 séries de resultados de parâmetros, como o PLD, energia natural afluente (ENA) e geração do SIN (CEPEL, 2013).

\subsubsection{Mecanismo de Realocação de Energia}

O MRE é um mecanismo financeiro, criado pelo Decreto $\mathrm{N}^{\mathrm{o}}$ 2.655/1998, para possibilitar a mitigação do risco hidrológico entre os agentes geradores. Portanto, o MRE assegura que todos os empreendimentos participantes recebam o montante energético proporcional a sua garantia física em relação a geração hidráulica total das usinas participantes do mecanismo.

O MRE abrange todos os empreendimentos hidráulicos com despacho centralizado, sendo facultativo a inclusão das PCHs, desde que sigam os procedimentos de comercialização específicos da Resolução Normativa $n^{\circ} 409$, 2010. É de responsabilidade do gerador, no caso de $\mathrm{PCH}$, integrar ou sair do mecanismo, excluso caso de perda de outorga, conforme Lei $\mathrm{n}^{\circ}$ 13.360, 2016.mouse

$\mathrm{O}$ processo de contabilização do MRE define o resultado individual mensal, por agente, todo final de mês. A Tarifa de Energia de Otimização (TEO) é destinada a compensação financeira dos empreendimentos participantes do MRE conforme Decreto $\mathrm{N}^{\circ} 2.655,1998$ e é disponibilizada pela ANEEL anualmente (CCEE, 2018).

A análise dos impactos do MRE demanda o estudo do Generation Scaling Factor (GSF), índice percentual que mede, em base mensal, a razão entre a geração total do MRE e a garantia física total das usinas do bloco.

\subsection{Conditional Value at Risk}


Conditional Value at Risk, ou simplesmente CVaR, é uma métrica de risco dada pela média das piores perdas esperadas durante um período de tempo sob determinado nível de confiança (Rockafellar and Uryasev, 2002), e pode ser definida, segundo Rockafellar and Uryasev (2002), de acordo com a seguinte equação:

$$
\begin{aligned}
& \text { CVaR }=\frac{1}{n S c e n} \sum_{s c=1}^{n S c e n} \text { Custo }_{s c}(x)- \\
& \frac{1}{\alpha * n S c e n} \sum_{s c=1}^{\alpha * n S c e n} \text { Custo }_{s c}(x),
\end{aligned}
$$

sendo $x$ a variação de contratação do portfólio, $\alpha$ as piores receitas e $n S c e n$ o número de cenários.

$\mathrm{O} C V \mathrm{R}$ é mais sensível à cenários críticos em relação à outra métricas de risco. É utilizado como estimador de riscos em avaliações financeiras que apresentam volatilidade de preços, como análises envolvendo projetos e investimentos de energias renováveis (Bruno et al., 2016) e mercado de energia (Sadeghi and Shavvalpour, 2006).

\section{METODOLOGIA}

O processo de avaliação do desempenho das PCHs, dentro e fora do MRE, foi realizado conforme fluxograma da Figura 1.

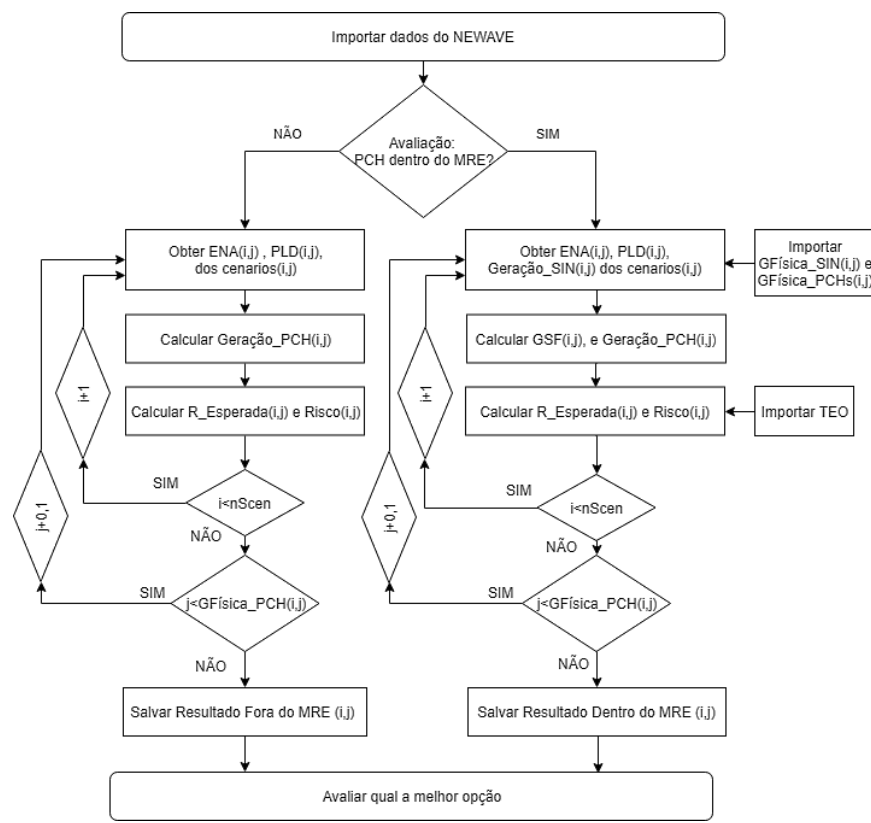

Figura 1 - Fluxograma de metodologia de cálculo do resultado das PCHs dentro e fora do MRE.

O algoritmo implementado é baseado na aplicação do CVaR e método de tomada de decisão com o objetivo de otimizar os cenários das séries de resultados financeiros das PCHs.

\subsection{Pequenas Centrais Hidroelétricas fora do Mecanismo de Realocação de Energia}

Para a análise do desempenho das PCHs fora do MRE, foram obtidas as projeções do PLD e da ENA do sudeste para o ano de estudo. As séries foram retiradas do resultado do deck do NEWAVE de setembro/2018.
A média de geração histórica das PCHs é a base da análise para o desempenho futuro de geração das usinas. A geração passada das usinas foi obtida dos relatórios de mercado disponibilizados pela CCEE referentes ao processo de contabilização, a partir do ano 2000, para uma amostra mais condizente com o comportamento de geração atual do SIN.

A geração média mensal foi obtida como uma média simples da geração histórica realizada nos meses correspondentes, dada pela equação a seguir:

$$
\text { GeraçãoMédia_PCH } H_{m}=\frac{\sum_{n} E g e r_{m}}{n},
$$

sendo GeraçãoMédia_PCH$H_{m}$ a geração média de energia elétrica calculada para a PCH no mês referido "m", Eger ${ }_{m}$ o montante de energia gerada no mês referido "m", registrado na CCEE, e $n$ a quantidade de meses considerados no estudo.

A geração futura mensal das PCHs foi obtida com a multiplicação da ENA do sudeste em relação à Média de Longo Termo (MLT), obtidas do modelo NEWAVE, pela geração média mensal das PCHs, conforme equação:

$$
\begin{aligned}
\text { Geração_PCH } & =\text { GeraçãoMédia_PCH } \\
& \times E N H_{-} M L T(\%)_{m},
\end{aligned}
$$

Sendo Geração_PCH $H_{m}$ a geração futura de energia elétrica da PCH no mês referido "m", ENA_MLT(\%) $m$ a energia natural afluente percentual do sudeste em relação à média de longo termo (MLT) no mês referido " $\mathrm{m}$ ".

O resultado financeiro das $\mathrm{PCHs}$ fora do MRE é obtido através da equação:

$$
\begin{aligned}
& \text { ReceitaForaMRE } E_{m}=\left(\text { Geração_PCH } H_{m}-\right. \\
& \text { Contratação } \left._{m}\right) \times \text { nHoras }_{m} \times P L D_{S E_{m}}+ \\
& \text { Contratação } o_{m} \times \text { PreçoContrato }_{m} \text {, }
\end{aligned}
$$

sendo ReceitaForaMRE $E_{m}$ o resultado financeiro da usina fora do MRE para o mês "m" de referência, Contratação $o_{m}$ o volume de energia contratado no mês "m", PreçoContrato $_{m}$ o preço de contrato no mês " $m$ " considerado como R $\$ 250$ / MWh, nHoras $_{m}$ o número total de horas no mês " $\mathrm{m}$ " referido e $P L D$ o PLD do sudeste no mês " $m$ " de referência.

\subsection{Pequenas Centrais Hidroelétricas dentro do Mecanismo de Realocação de Energia}

Para a análise do desempenho das PCHs dentro do MRE, além da geração futura das mesmas, foram obtidas as suas respectivas garantias físicas, sem sazonalização, para o ano de estudo, através dos relatórios de mercado da CCEE. Foram obtidas as garantias físicas de 2,99 MW para a PCH I, 4,42 MW para a PCH II, 7MW para a PCH III e 19,99MW para a PCH IV.

A geração do SIN foi obtida do resultado do deck do NEWAVE e a garantia física do SIN obtida através dos relatórios de mercado da CCEE e das premissas de entrada de novos empreendimentos no SIN. Também foi incluída na simulação a TEO de dezembro/2018, fixada à R\$ 11,87/MWh 
para todos os cenários. O cálculo da TEO é destinado à cobertura dos custos incrementais de O\&M das usinas hidrelétricas, o que torna a mesma de difícil previsão. Portanto, foi considerada a TEO vigente, consideração que simplifica a simulação e pouco afeta os resultados do estudo.

O GSF foi obtido através da fórmula:

$$
G S F_{m}=\frac{G e r a c ̧ a ̃ o \_S I N}{m},
$$

sendo $G_{S} F_{m}$ o GSF do MRE no referido mês "m", Geração_SIN ${ }_{m}$ o resultado de geração do SIN no mês de referência "m", considerando também a média histórica da geração de pequenos empreendimentos, e GFísica_SIN $N_{m}$ a garantia física total do SIN no mês "m" de referência.

$\mathrm{O}$ resultado financeiro das $\mathrm{PCH}$ dentro do MRE é obtido através da equação:

$$
\begin{aligned}
& \text { ReceitaDentroMRE } E_{m}=\left[\left(\text { GFísica_PCH } H_{m} \times\right.\right. \\
& \left.\left.G S F_{m}\right)- \text { Contratação }_{m}\right] \times \text { nHoras }_{m} \times \\
& P L D \_S E_{m}+\left[\text { Geração_PCH } H_{m}-\left(\text { GFísica_PCH } H_{m} \times\right.\right. \\
& \text { GSF)] } \times \text { nHoras }_{m} \times \text { TEO }_{m}+\left(\text { Contratação }_{m} \times\right. \\
& \text { nHoras } \left._{m} \times \text { PreçoContrato }_{m}\right) \text {, }
\end{aligned}
$$

sendo ReceitaDentroMRE $E_{m}$ o resultado financeiro da $\mathrm{PCH}$ para o mês "m" de referência, GFísica_ $P C H_{m}$ a garantia física da PCH no mês referido "m" e $T E O_{m}$ a tarifa de energia de otimização.

\section{EXPERIMENTO E RESULTADOS}

Foram calculadas as receitas individuais mensais das PCHs, conforme (4), liquidando todo o resultado de geração das mesmas no MCP. A Figura 2 apresenta a distribuição de frequência das 2000 séries de retorno anual financeiro das usinas para o ano de 2019.
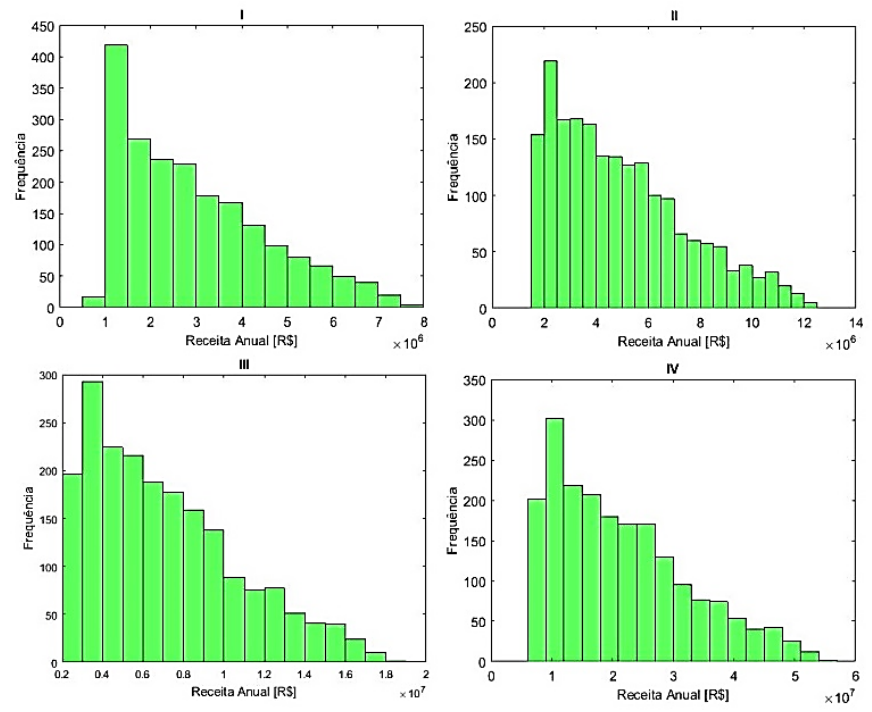

Figura 2 - Distribuição de Frequência das Receitas das PCHs I, II, III e IV fora do MRE.
A PCH I apresenta probabilidade de $58 \%$ de um retorno variável de 1 a 3 milhões de reais de receita; a PCH II apresenta $44 \%$ de probabilidade de receita até 4 milhões; a PCH III apresenta $46 \%$ de probabilidade de receita de 2 a 6 milhões; a PCH IV apresenta $36 \%$ de probabilidade de receita de 10 a 20 milhões. O resultado financeiro esperado, obtido através da soma das médias das 2000 séries de receitas anuais das 4 PCHs, totalizaram aproximadamente 36,4 milhões de reais. A receita em risco anual das $4 \mathrm{PCHs}$, calculada com a métrica de risco $\mathrm{CVaR}$, na qual foram desconsideradas a média dos $5 \%$ piores resultados de receita anual de cada $\mathrm{PCH}$, totalizou $\mathrm{R} \$ 23,4$ Milhões.

Para a simulação das PCHs dentro do MRE, conforme (6), foram consideradas as séries de maior ocorrência para estimar a margem de receita para o agente gerador. A Figura 3 apresenta a distribuição de frequência das séries de resultados financeiros das usinas dentro do MRE, no caso das usinas totalmente descontratadas.
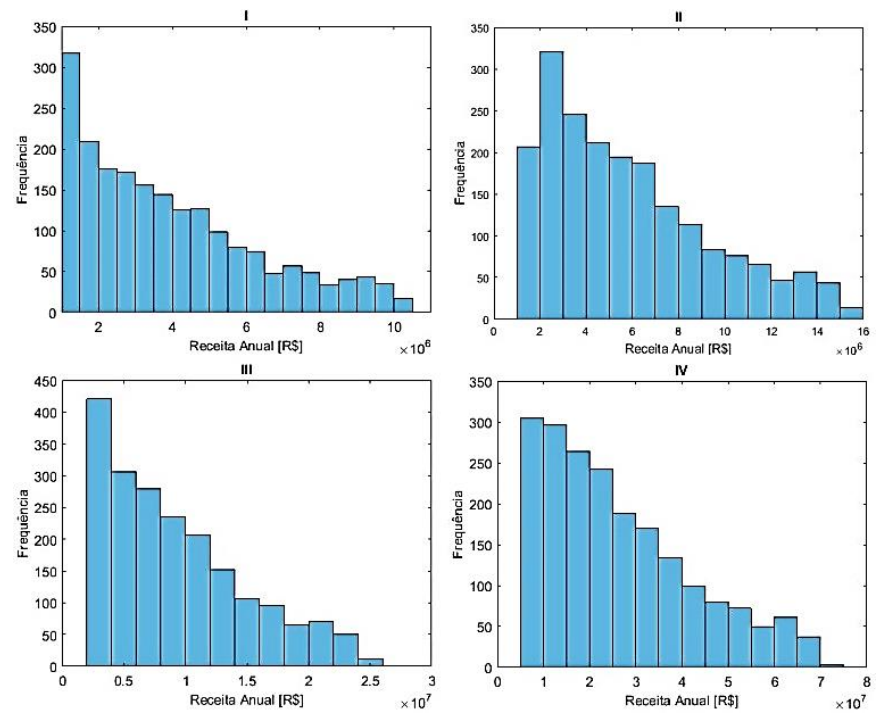

Figura 3 - Distribuição de Frequência das Receitas das PCHs I, II, III e IV dentro do MRE.

Cerca de $44 \%$ das séries de receita da PCH I estão entre o intervalo de 1 a 3 milhões; a PCH II apresenta $49 \%$ de probabilidade de receita até 4 milhões; a PCH III apresenta $50 \%$ de probabilidade de receita até 8 milhões e a PCH IV apresenta $40 \%$ das séries entre 10 e $\mathrm{R} \$ 25$ milhões. A receita esperada anual de cada usina, calculada a partir da média das 2000 séries dos resultados financeiros, resultaram em aproximadamente 45,5 milhões de receita anual total. Considerando a métrica de risco CVAR, eliminando a média dos 100 piores resultados, a receita em risco é de aproximadamente 32,8 milhões para o somatório das usinas.

\subsection{Perfil de Risco das PCHS}

Os empreendimentos apresentam perfis de risco com diferentes configurações para cada volume de contratação. Os gráficos da Figura 4 demonstram o perfil de risco das PCHs descontratadas, ao longo do ano de 2019, referenciando o PLD 
médio anual e as receitas anuais atreladas aos mesmos. As séries do CVaR estão indicadas pelos pontos em vermelho nos gráficos.

Para todas as PCHs (Figura 4), a participação no MRE garante redução na dispersão das séries, sendo que a faixa de resultado financeiro admissível para cada PLD é mais estreita, aumentando a previsibilidade do retorno financeiro dos empreendimentos. A inclinação da curva de resultados também aumenta, convergindo para uma melhora da receita na maioria dos cenários de PLD.

Pode-se concluir que em todos os cenários de PLD, os resultados dentro do MRE estão apresentando um retorno financeiro maior ao agente gerador, o que demonstra a maximização do retorno financeiro.
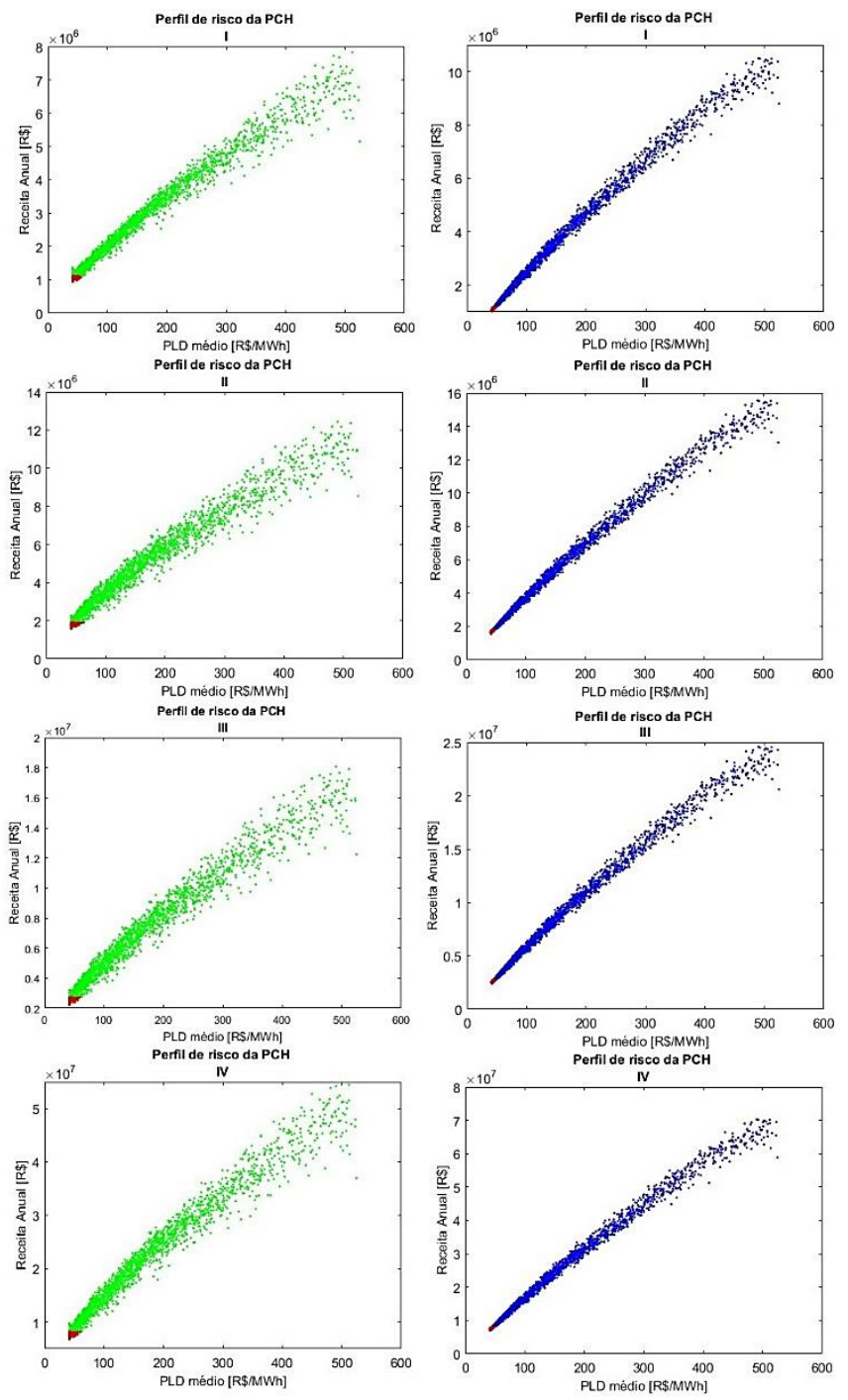

Figura 4 - Perfil de Risco das PCHs.

\subsection{Análise do Portfólio das PCHs}

Nos gráficos da Figura 5 pode-se observar as receitas em risco e as receitas esperadas para diferentes valores de contratação para todas as PCHs, individualmente, dentro e fora do MRE.
As curvas de receitas esperadas das $\mathrm{PCHs}$ foram obtidas através da aplicação das equações da Seção 3. E as receitas em risco, foram calculadas através de (1). Sendo que, para cada valor de contração das usinas, é possível avaliar simultaneamente a receita e o risco atrelado às mesmas em determinado cenário. Consequentemente, através do estudo das curvas, pode-se chegar à otimização da contratação dentro e fora do MRE, considerando como critério de tomada de decisão, o cenário de menor risco atrelado à melhor receita esperada.

Para a simulação dos cenários de contratação, foi fixado um valor de contrato de $\mathrm{R} \$ 250,00 / \mathrm{MWh}$, baseada em um preço médio de mercado e uma taxa de variação de $0,1 \mathrm{MWm}$ por cenário, para obtenção das curvas de risco intrínseco individuais das PCHs.

Para a PCH I, fora do MRE, conforme Figura 5, o melhor cenário de contratação seria 1,3 MWm, ponto de menor valor da curva verde pontilhada, que representa a receita em risco do gerador. Dentro do MRE, o ponto ótimo de contratação seria 2,2 MWm, posição na qual tem-se a menor receita em risco, representada pelo menor ponto da curva pontilhada azul na Figura 5. Pode-se observar que acima destas contratações, o agente está mais exposto às variações do preço do mercado spot e do GSF.
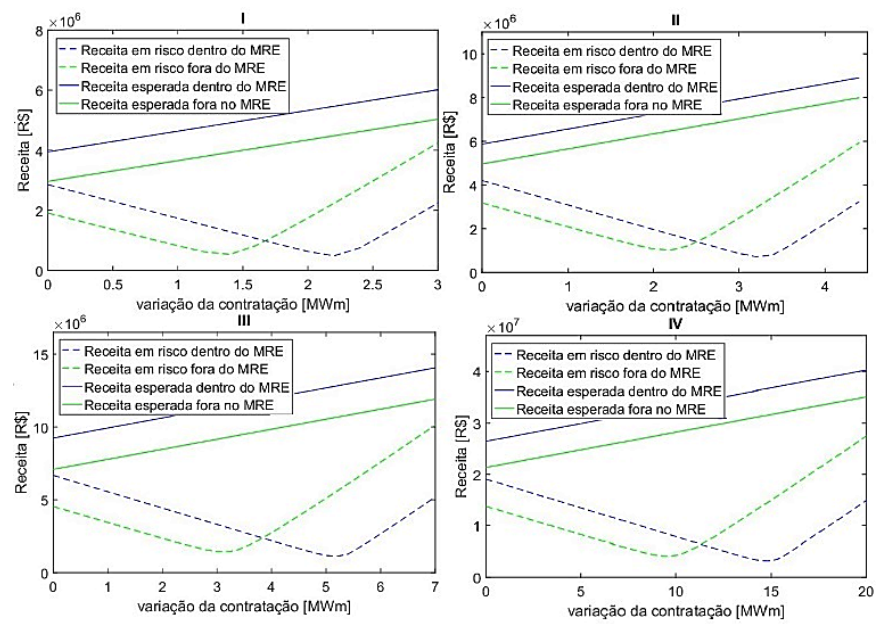

Figura 5 - Curvas de Risco Intrínseco das PCHs dentro e fora do MRE.

Para a PCH II, conforme Figura 5, o melhor cenário de contratação fora do MRE seria 2,2 MWm, onde o gerador assumiria uma receita de menor risco. Dentro do MRE, o ponto ótimo de contratação seria 3,3 MWm, posição na qual tem-se a menor receita em risco. Caso o agente aumentasse sua contratação, em ambos os casos estaria assumindo um risco maior de resultado em sua receita.

Para a PCH III, indicada na Figura 5, o melhor cenário de contratação fora do MRE seria 3,2 MWm, no qual a receita em risco é menor. Dentro do MRE, o ponto ótimo de contratação da PCH seria 5,2 MWm. Caso o agente optasse por um risco maior, poderia aumentar sua contratação, até o limite de sua garantia física. 
No caso da PCH IV (Figura 5), dentro do MRE, a melhor contratação seria $15 \mathrm{MWm}$, ponto mínimo da curva pontilhada azul. Para contratações abaixo de 11,8 MWm, caso a usina estivesse no mecanismo, apesar da receita esperada ser superior à receita fora do $\mathrm{MRE}$, a receita em risco seria maior. Se a $\mathrm{PCH}$ estivesse fora do MRE, o melhor cenário de contratação seria de $10 \mathrm{MWm}$, onde teria a melhor relação entre a receita esperada e o menor risco.

\section{CONCLUSÕES}

Este trabalho foi realizado com foco na análise dos resultados da participação das PCHs no MRE, com aplicação de métrica de risco CVaR e variação da contratação para maximização do portfólio de energia do gerador.

Para todas as PCHs, o retorno financeiro anual ao agente gerador foi maior ao alocar seus empreendimentos para dentro do MRE. Apesar de em alguns meses o mecanismo apresentar uma diminuição na receita de certas PCHs, a compensação do resultado de geração no restante do ano é superior à perda de receita desses meses. O MRE se tornou, então, eficaz no compartilhamento dos riscos hidrológicos entre as usinas em estudo, não só atenuando os riscos de geração individual, mas também melhorando a receita do agente.

Considerando o resultado financeiro médio anual individual proporcionado pela entrada no MRE, a receita foi de 18 a $33 \%$ maior, tendo-se como referência a receita média anual esperada das PCHs, caso o gerador não compartilhasse os riscos hidrológicos com as outras usinas do SIN, estando fora do MRE.

Quanto ao portfólio de contratação das PCHs, foi possível analisar que na maioria dos casos, estando parcialmente contratada, os lucros das usinas foram maximizados, contanto que estivessem na margem de menor receita em risco. O cenário ideal foi a contratação de 70 a $75 \%$ da garantia física das PCHs dentro do MRE. Neste caso, deve-se avaliar o preço de contrato em relação aos preços de mercado, buscando o volume ótimo a ser contratado.

A análise demonstrou também que o MRE pode não ser tão vantajoso para usinas que possuem um bom desempenho de geração, principalmente nos casos de GSF elevado, pois a usina estaria deixando de liquidar sua energia à preços mais elevados de PLD. Ressalta-se, porém, que essa opção só é válida para as $\mathrm{PCHs}$, que não possuem obrigatoriedade em participar do mecanismo.

O objetivo principal do MRE, entretanto, tem sido desestabilizado pelas incertezas de despacho das fontes intermitentes não hidráulicas que tem se agregado ao sistema e impactado o despacho das usinas hidrelétricas. Além disso, os cenários de crise hídrica podem alavancar um baixo desempenho de geração do bloco e afetar a receita das usinas participantes, além do esperado. Todas essas inseguranças tornam a análise do MRE mais complexa e a decisão do gerador em participar ou não, mais difícil. Uma solução pode ser a contratação de parte da garantia física da usina para atenuar o risco e proteger o gerador de cenários de maior exposição. Apesar de todas essas complicações, o MRE ainda demonstrou ser eficiente como um maximizador de receita e atenuador de riscos hidrológicos para as PCHs em estudo.

As discussões atuais sobre o MRE envolvem questões judiciais e os interesses dos geradores em melhor mitigar seus riscos com o mercado. Neste âmbito, foi aberta pela ANEEL a Audiência Pública nº 24/2017 como forma de obter subsídios para o aprimoramento da proposta de revisão da Resolução Normativa 409/2010, buscando adequar a resolução à legislação vigente.

Como proposta de continuidade deste trabalho, sugere-se a análise da sazonalização da garantia física das $\mathrm{PCHs}$ perante os cenários do MRE, o estudo da variação da contratação das usinas considerando a sazonalização da garantia física das mesmas e a possível contribuição à Audiência Pública $n^{\circ}$ 24/2017, que propõe a revisão dos critérios de participação dos empreendimentos não despachados centralizadamente no MRE.

\section{REFERÊNCIAS}

ANEEL (2010) RESOLUÇÃO NORMATIVA No 409, DE 23/08/2010. Available at: http://www2.aneel.gov.br/cedoc/ren2010409.pdf.

ANEEL (2003) RESOLUÇÃO Nº 652 DE 9 DE DEZEMBRO DE 2003. Available at: http://www.aneel.gov.br/cedoc/res2003652.pdf.

Bruno, S. et al. (2016) 'Risk neutral and risk averse approaches to multistage renewable investment planning under uncertainty', European Journal of Operational Research. Elsevier B.V., 250(3), pp. 979-989. doi: 10.1016/j.ejor.2015.10.013.

CCEE (2018) '04 - Mecanismo de Realocação de Energia ( MRE )', pp. 1-58. Available at: https://www.ccee.org.br/ccee/documentos/CCEE_07615 9.

CEPEL, E.- (2013) 'Modelo Estratégico de Geração Hidrotérmica a Subsistemas Equivalentes Interligados NEWAVE, Manual do Usuário'. Available at: http://www2.aneel.gov.br/aplicacoes/audiencia/arquivo/2 013/086/documento/manualusuario.pdf.

DECRETO No 2.655, DE 2 DE JULHO DE 1998 (1998). Available at: http://www2.camara.leg.br/legin/fed/decret/1998/decreto -2655-2-julho-1998-361411-normaatualizada-pe.html.

EPE (2015) 'Plano Decenal de Expansão de Energia 2024. Ministério de Minas e Energia. Empresa de Pesquisa Energética - EPE'. Available at: epe.gov.br/PDEE/Relatório Final do PDE 2024.pdf.

Fanzeres, B., Street, A. and Barroso, L. A. (2015) 'Contracting Strategies for Renewable Generators: A Hybrid Stochastic and Robust Optimization Approach', IEEE Transactions on Power Systems. Institute of Electrical and Electronics Engineers Inc., 30(4), pp. 1825-1837. doi: 10.1109/TPWRS.2014.2346988.

LEI No 13.360, DE 17 DE NOVEMBRO DE 2016. Disponível em: http://www.planalto.gov.br/ccivil_03/_ato20152018/2016/lei/L13360.htm 
Masukawa, F. T. (2011) Avaliação de critérios de risco na negociação de contratos bilaterais de energia em Centrais Hidrelétricas de Pequeno Porte - PCH. Dissertação (Mestrado) Universidade de Brasília.

Moita, R. M. S. (2008) 'Entry and externality: Hydroelectric generators in Brazil', International Journal of Industrial Organization. doi: 10.1016/j.ijindorg.2008.02.006.

ONS (2019) O Sistema Interligado Nacional. Available at: http://ons.org.br/paginas/sobre-o-sin/o-que-e-o-sin (Accessed: 15 April 2019).

Rockafellar, R. T. and Uryasev, S. (2002) 'Conditional valueat-risk for general loss distributions', Journal of Banking and Finance, 26(7), pp. 1443-1471. doi: 10.1016/S03784266(02)00271-6.

Sadeghi, M. and Shavvalpour, S. (2006) 'Energy risk management and value at risk modeling', Energy Policy. doi: 10.1016/j.enpol.2005.07.004.

Street, A. et al. (2009) 'Risk constrained portfolio selection of renewable sources in hydrothermal electricity markets', IEEE Transactions on Power Systems, 24(3), pp. 11361144. doi: 10.1109/TPWRS.2009.2022981. 